\title{
HIGH ALTITUDE ADAPTATION AND ANAEROBIOSIS IN SCELOPORINE LIZARDS
}

\author{
Albert F. BennetT* And John Ruben \\ Department of Zoology, University of California, Berkeley, California 94720, U.S.A.
}

(Received 4 October 1973)

\begin{abstract}
The duration of maximal activity and the intensity of anaerobic metabolism were measured in lowland Sceloporus occidentalis at low (200 ft) and high $(10,150 \mathrm{ft})$ elevations and compared to those of resident $S$. occidentalis and $S$. graciosus at high altitude.

2. The duration of maximal activity is independent of altitude: all groups of S. occidentalis had the same stamina, regardless of the altitude of experimentation or prior residence.

3. The amount of lactate formed during activity is also independent of altitude or prior residence.

4. No indications of altitude adaptation were found in these species. It is hypothesized that the basically anaerobic activity patterns of small lizards constitute a preadaptation to colonization of high altitudes.
\end{abstract}

\section{INTRODUCTION}

Mammals and birds inhabiting high altitudes demonstrate a suite of compensatory physiological adjustments facilitating the acquisition of oxygen. Such hematological adaptations as greater hematocrit, oxygen capacity and oxygen affinity are commonly associated with the lower levels of environmental oxygen available to highland animals. Similar adaptations have yet to be demonstrated unequivocally in reptiles from high altitudes. A compensatory increment in hematological parameters has been reported in some reptiles exposed naturally or artificially to high altitude (Richter, 1933; Hadley \& Burns, 1968; Vinegar \& Hillyard, 1972; Weathers \& McGrath, 1972). Other investigators, often examining the same species, have not found such compensation (Sokolov, 1941; Altland \& Parker, 1955; Dawson \& Poulson, 1962; Pough, 1969; Weathers \& White, 1972). A higher rate of resting oxygen consumption ( $+30-45$ per cent) has been reported in highland vs. lowland forms of sceloporine lizards (Bartlett, 1970). The significance, indeed the adaptive value, of such an increased metabolic rate is unknown. At present, we can only conclude that adaptations to sustain aerobiosis at high altitudes, if they exist at all, are not as widespread or as well developed in reptiles as in the homeothermic groups.

Even if hematological adaptations promoting increased aerobiosis do exist in highland reptiles, whether they impart more stamina under oxygen stress, e.g. during activity, has never been examined. It is unknown whether highland animals perform better in hypoxic situations than do lowland forms.

* Present address: School of Biological Sciences, University of California, Irvine, California 92664.
Increased stamina could result from either greater aerobic or greater anaerobic capabilities. Evidence has accumulated that many lowland reptiles during maximal activity are largely dependent upon and tolerant of anaerobic metabolism (Moberly, 1968a, b; Bennett \& Licht, 1972; Bennett \& Dawson, 1972; Gatten, 1973): Lactic acid production provides most of the energy generation during rapid activity. The dynamics of lactate production have not been examined in reptiles resident at high altitude or in lowland animals during hypoxia. These experiments were undertaken to determine whether there is any difference in activity performance in low and high altitude reptiles and whether the patterns of anaerobic metabolism are similar in these forms.

Lizards of the genus Sceloporus in the western United States present an excellent altitudinal series for comparative studies. Sceloporus occidentalis, the western fence lizard, occupies an altitudinal range from sea level to over $9000 \mathrm{ft}$ in the mountains of California. A sympatric species, $S$. graciosus, is more commonly found at high elevations than is $S$. occidentalis and it ranges even higher $(10,000+\mathrm{ft})$ (Stebbins, 1954). We measured maximal activity and anaerobic metabolism in lowland $S$. occidentalis in the lowlands and transported a lowland group to high altitude. Here the experiments were repeated along with those on resident populations of $S$. occidentalis and $S$. graciosus.

\section{MATERIALS AND METHODS}

Lowland Sceloporus occidentalis (mean wt., 12.0 g) were collected near Napa, Napa County, California (elevation approximately $1000 \mathrm{ft}$ ). Highland $S$. occidentalis $(16.2 \mathrm{~g})$ and $S$. graciosus $(5.8 \mathrm{~g})$ were collected at $8000-9000 \mathrm{ft}$ in the White Mountains, Inyo County, California. Experimentation was performed at the University of California, Berkeley (elevation $230 \mathrm{ft}$ ), for 
lowland determinations and at Crooked Creek Laboratory, White Mountain Research Station of the University of California (elevation 10,150 ft). Animals were utilized 24-48 hr after capture. Lowland $S$. occidentalis were exposed to highland conditions for less than $24 \mathrm{hr}$ before experimentation. Lowland animals were measured in both the lowlands and highlands; highland animals were measured only in the highlands. All experiments were carried out in June during the normal season of activity.

Anaerobic metabolism during maximal activity was measured according to the method of Bennett \& Licht (1972). Lizards were weighed and placed overnight in the dark at $35^{\circ} \mathrm{C}$, a temperature within the activity range of both species (Brattstrom, 1965). The following morning, some animals were removed and exercised for $30 \mathrm{sec}$, $90 \mathrm{sec}$ or $5 \mathrm{~min}$, and others were left unstimulated as

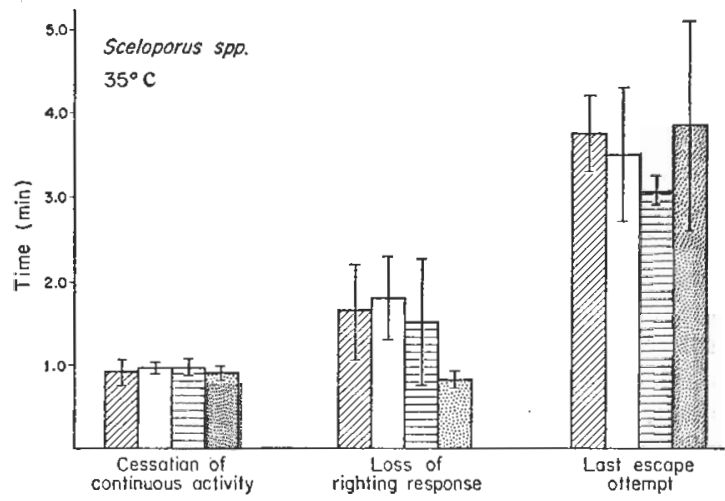

Fig. 1. Stamina of sceloporine lizards during maximal activity at different altitudes. The height of the wide vertical bars indicates the mean duration of activity cessation; 95 per cent confidence limits of the means are given by narrow vertical bars. Experimental groups consisted of three to ten animals. Groups are indicated as follows: bar with diagonal lines, lowland $S$. occidentalis exercised in lowlands (200 ft); open bar, lowland $S$. occidentalis in highlands $(10,150 \mathrm{ft})$; bar with horizontal lines, highland $S$. occidentalis in highlands; stippled bar, highland $S$. graciosus in highlands. resting controls. When released from their containers, the lizards ran at high speeds around an activity chamber. This initial activity required no stimulation. Later activity was elicited by prodding the animal with bluntnosed forceps about the hind legs and tail. The cessation of spontaneous activity, the abolition of the righting response and total exhaustion were timed with a stopwatch. At the end of the activity period, the animals were decapitated and homogenized in $0.6 \mathrm{~N}$ perchloric acid. Lactate content of the homogenates was later determined by an enzymatic kit (see Bennett \& Licht, 1972, for methodology).

Statistical differences among groups were tested by Kruskal-Wallis tests, using the 0.05 probability level of hypothesis rejection. If differences among groups were indicated, pairwise comparisons between the groups were made by Mann-Whitney tests, using the 0.01 probability level.

\section{RESULTS}

Activity proceeded in a well-defined pattern. When released from their container, the lizards ran rapidly and continuously around the activity chamber. This running included jumping, rapid reversal of direction and flipping of the entire body. If the animals had not been confined, capturing them during this period would have been difficult. The lizards righted themselves rapidly $(<0.5 \mathrm{sec})$ when placed on their backs. This active phase, during which little stimulation was required to elicit activity, lasted approximately $1 \mathrm{~min}$. This was followed by a period of infrequent walking only when externally stimulated. The animals would move $0.5-1.0 \mathrm{ft}$ and stop; they were visibly fatigued. The righting response disappeared about $90 \mathrm{sec}$ after the initiation of activity. The last escape attempt was made between 3 and 4 min after activity began; no stimulation could provoke the lizards to move further.

The average times of continuous activity, loss of righting response and total exhaustion for all

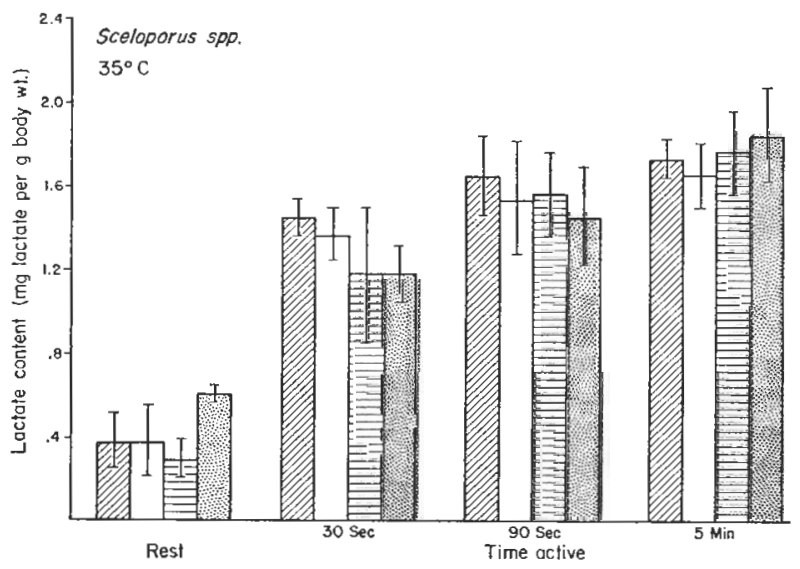

Fig. 2. Lactate content during rest and after activity in sceloporine lizards at different altitudes. Symbol definitions are given in Fig. 1. 
experimental groups are given in Fig. 1. The duration of continuous activity was measured as the time at which the lizard first failed to respond to a touch stimulus. The loss of righting response was the first time the animal failed to turn over when placed on its back. Time to total exhaustion was the time of last escape response from stimulation. With one minor exception, there is no significant difference among groups or species within any activity category: stamina is identical regardless of the site of experimentation or origin of animals. The sole exception is that $S$. graciosus loses its righting response before any of the groups of $S$. occidentalis.

The determinations of lactate formation encompass all phases of activity. The 30 -sec measurement reflects the maximum rate of anaerobic energy generation (anaerobic scope); $90 \mathrm{sec}$, the effective amount of anaerobic energy which could be mobilized in an attempted escape; and $5 \mathrm{~min}$, the total amount of lactate formed and tolerated at exhaustion. Lactate contents of different experimental groups are reported in Fig. 2. There are no significant differences among the three experimental groups of $S$. occidentalis within any activity state: lactate content is only influenced by the amount of activity, not the original altitude of the animals or the site of experimentation. Resting levels of lactate are higher in $S$. graciosus than in any of the groups of $S$. occidentalis, indicating the former animals were probably more active in their containers overnight. Lactate contents of $S$. occidentalis measured in the lowlands after $30 \mathrm{sec}$ of activity are significantly higher than in S. graciosus. No interspecific differences in lactate content occur after $90 \mathrm{sec}$ or $5 \mathrm{~min}$ activity.

\section{DISCUSSION}

The data on type and duration of activity fail to show any altitude effect or adaptation to high altitude within this group of sceloporine lizards. Lowland $S$. occidentalis have just as much endurance and qualitatively identical activity at both 200 and $10,150 \mathrm{ft}$, even though oxygen pressure at the latter altitude is only two-thirds that at the former. They have as much stamina as high-altitude residents of the same species and of $S$. graciosus, a species generally associated with high altitudes. The fact that behavior of all these groups is essentially identical suggests that even if aerobic adaptations are present in high altitude residents, they are not of significance in prolonging activity or increasing stamina.

It might have been hypothesized a priori that high altitude residents, chronically exposed to lower levels of oxygen, might have a greater tolerance of lactic acid and anaerobic metabolism. The present experiments show that this is not the case in the species examined here. Lactate production during activity and maximal levels at exhaustion are no greater in high altitude residents. Anaerobic scope is less in $S$. graciosus, a high altitude species, than in $S$. occidentalis from the lowlands. The anaerobic scope of $S$. occidentalis (1.76-2.14 mg lactate/g body weight per min-see Fig. 2) is greater than those previously reported for most other species of small lizards; levels of lactate tolerated at exhaustion are also greater (Bennett \& Licht, 1972). These capacities may be factors involved in its colonization of high altitudes.

Although the sceloporine lizards have a wide altitudinal distribution, they do not occur at very high altitudes. It is possible that other high-altitude saurian species may have evolved anaerobic adaptations to hypoxia. A group of particular interest is the South American iguanid genus Liolaemus, species of which inhabit altitudes up to $17,000 \mathrm{ft}$. Observations (Bennett, unpublished data) on Liolaemus mocquardi from $14,500 \mathrm{ft}$ indicate that this species is still active and aggressive when its lactate concentrations are greater than those of $S$. occidentalis at exhaustion. These capacities are, however, no higher than those of some lowland forms (e.g. Dipsosaurus dorsalis; Bennett \& Licht, 1972). Further examinations of other species of Liolaemus, including lowland forms, are necessary to evaluate anaerobic adaptations within this group.

The data presented in this paper suggest that there has been no significant adaptation to high altitude in these species of sceloporine lizards. In a sense, they are already adapted to life at high altitudes by their metabolic patterns. Since they are highly dependent upon anaerobic metabolism during activity, they are largely independent of external oxygen sources. Further research should be directed into an examination of organismal aerobic adaptations: the levels of aerobic scope and aerobic work output. These may show adaptations of high altitude animals at nonmaximal activity levels. Measurements of oxygen debt would indicate whether recovery from activity is more rapid in high-altitude residents. It may well be, however, that reliance upon and tolerance of anaerobiosis constitutes the major factor in permitting colonization of high altitudes by reptiles.

Acknowledgements-Support for this study was provided by a Miller Postdoctoral Research Fellowship to A. F. B. and N.S.F. Grant No. 22642 to Paul Licht. We thank the staff of the White Mountain Research Laboratory for their courteous assistance in facilitating our study and Paul Licht for reading and criticizing the manuscript.

\section{REFERENCES}

Altland P. D. \& Parker M. (1955) Effects of hypoxia upon the box turtle. Am. J. Physiol. 180, 421-427.

Bartzett P. N. (1970) Seasonal and elevational comparisons of oxygen consumption rates in the lizard Sceloporus occidentalis. Ph.D. dissertation, University of California, Riverside. 
Bennetr A. F. \& Dawson W. R. (1972) Aerobic and anaerobic metabolism during activity in the lizard Dipsosaurus dorsalis. J. comp. Physiol. 81, 289-299.

BenNetT A. F. \& Lichi P. (1972) Anerobic metabolism during activity in lizards. J. comp. Physiol. 81, 277288.

Brattstrom B. H. (1965) Body temperatures of reptiles. Am. Midl. Nat. 73, 376-422

Dawson W R. \& Poulson T. L. (1962) Oxygen capacity of lizard bloods. Am. Midl. Nat. 68, 154-164.

Gatten R. E., JR. (1973) Aerobic and anaerobic metabolism during activity in the turtles Pseudemys scripta and Terrapene ornata. Ph.D. dissertation, University of Michigan, Ann Arbor.

Hadley N. F. \& Burns T. A. (1968) Intraspecific comparisons of the blood properties of the sideblotched lizard, Uta stansburiana. Copeia 1968, 737740.

MOBeRLy W. R. (1968a) The metabolic responses of the common iguana, Iguana iguana, to activity under restraint. Comp. Biochem. Physiol. 27, 1-20.

MoberLy W. R. (1968b) The metabolic responses of the common iguana, Iguana iguana, to walking and diving. Comp. Biochem. Physiol. 27, 21-32.

Pough F. H. (1969) Environmental adaptations in the blood of lizards. Comp. Biochem. Physiol. 31, 885-901.
RIChTer R. (1933) Die Wirkung einiger klimatischen Faktoren sowie der jahreszeitlichen Periodizität auf den Erythrocytenspiegel europäischer Reptilien. Zool. Jahrb. 52, 433-450.

Sokolov A. N. (1941) Change in blood composition and the role of blood storage sites in decreased atmospheric pressure in cold-blooded animals. Med. Zh. (Kiev) 11, 145-157. (In Ukranian; Russian and German summaries.)

Stebins R. C. (1954) Amphibians and Reptiles of Western North America, pp. 240-244. McGraw-Hill, New York.

Vinegar A. \& Hillyard S. D. (1972) The effects of altitude on oxygen-binding parameters of the blood of the iguanid lizards, Sceloporus jarrovi and Sceloporus occidentalis. Comp. Biochem. Physiol. 43A, 317-320.

Weathers W. W. \& McGrath J. J. (1972) Acclimation to simulated altitude in the lizard Dipsosaurus dorsalis. Comp. Biochem. Physiol. 42A, 263-268.

Weathers W. W. \& White F. N. (1972) Hematological observations on populations of the lizard Sceloporus occidentalis from sea level and altitude. Herpetologica 28, 172-175

Key Word Index-Activity; altitude; anaerobiosis; exercise; lactic acid; lizard; reptile; Sceloporus. 